Pacific Journal of Mathematics

UN THÉORÈME DU GENRE “ANDÔ-EDWARDS”, POUR LES 


\section{UN THEOREME DU GENRE “ANDÔ-EDWARDS” POUR LES FRÉCHET ORDONNÉS NORMAUX}

\section{Alain Goullet De Rugy}

Let $E$ be an ordered locally convex topological vector space whose positive cone is normal, closed and generating. It is an important problem to characterize those spaces $E$, whose topological dual $E^{\prime}$ is a lattice for the dual ordering. It is proved here, with the use of Choquet's theory of weakly complete cones, that if $E$ is a Fréchet space, $E^{\prime}$ is lattice if and only if $E$ has the Riesz decomposition property. In fact, a stronger result is proved which is, even in the Banach case, an improvement of the classical Andô's theorem. An application to the duality of order ideals is given.

Il est bien connu que si $E$ vérifie la propriété d'interpolation de Riesz:

Si $u, v, u^{\prime}, v^{\prime} \in E$ sont tels que: $u, v \leqq u^{\prime}, v^{\prime}$, il existe $w \in E$ tel que:

$$
u, v \leqq w \leqq u^{\prime}, v^{\prime}
$$

qui est équivalente au "lemme de décomposition de Riesz", $E$ ' est réticulé. On se propose d'examiner la réciproque lorsque $E$ est un Fréchet. Plus précisement, considérons l'énoncé suivant ("théorème d'extension avec contraintes"):

Enoncé. On suppose que $E^{\prime}$ est réticulé. Soient $F$ une face faiblement fermée de $E_{+}^{\prime}, 1$ une fonction numérique additive, positivement homogène et continue sur $F$, et $u, v, u^{\prime}, v^{\prime}$ quatre éléments de $E$ tels que:

$$
u, v \leqq u^{\prime}, v^{\prime}
$$

et

$$
u(x), v(x) \leqq \underline{l}(x) \leqq u^{\prime}(x), v^{\prime}(x)
$$

Alors, il existe un élément $w$ de $E$ tel que:

$$
u, v \leqq w \leqq u^{\prime}, v^{\prime}
$$

et

$$
w(x)=\underline{l}(x)
$$

$(\forall x \in F)$.

(Dans cet énoncé, comme dans tout ce travail, on identifie un élément de $E$ et la forme linéaire faiblement continue sur $E^{\prime}$ corre- 
spondante). Lorsque $E$ est un Banach et $F=\{0\}$, cet énoncé est le théorème d'Andô ([1], Theorem 2). Lorsque $E$ est un Banach dont la norme est définie à partir d'une unité d'ordre et $F$ quelconque, cet énoncé est le corollaire le plus important du théorème d'Edwards de séparation de fonctions convexes sur un simplexe ([4]). Le but $\mathrm{du}$ présent travail est de montrer (Th. 3.7 et corollaires) que cet énoncé est vrai lorsque $E$ un Fréchet. Pour $F=\{0\}$, il donne la réciproque annoncée.

La notion de cône faiblement complet joue un rôle essentiel dans ce travail. C'est elle qui permet d'obtenir le résultat annoncé tout en conservant, dans la démonstration, les technique utilisées par Asimow et Ellis pour le montrer lorsque $E$ est un Banach et $F=\{0\}$.

1. Preliminaires. Les cônes faiblement complets.

Notations 1.1. Soient $E$ un e.l.c. faible (c'est-à-dire un espace dont la topologie n'est autre que $\sigma\left(E, E^{\prime}\right)$ ) et $X$ un cône convexe saillant complet de $E$. Par abus de langage, on dira qu'une fonction positivement homogène et additive sur $X$ et à valeurs dans un espace vectoriel quelconque est une fonction linéaire sur $X$. On note $L_{s}(X)$ (resp. $L_{c}(X)$ ) l'espace des formes linéaires et s.c.s. (resp. continues) sur $X$. Si $f$ est une fonction numérique sur $X$, majorée (resp. minorée) par un $l \in L_{c}(X)$, on pose:

$$
\left.\left.\widehat{f}=\inf \left\{l \in L_{c}\right) X\right): l \geqq f\right\},
$$

(resp.:

$$
\left.\check{f}=\sup \left\{l \in L_{c}(X): l \leqq f\right\} .\right)
$$

L'opération "^" tire son principal intérêt des résultats suivants (pour les démonstrations, voir [5], 4.10, 4.12 et 4.13):

Proposition 1.2. Soient $l_{1}, \cdots, l_{n} \in L_{s}(X)$ et $f=\sup \left(l_{1}, \cdots, l_{n}\right)$. Alors, (a) Pour tout $x \in X$, il existe $x_{1}, \cdots, x_{n} \in X$ tels que:

$$
x=\sum_{1}^{n} x_{i} \quad \text { et } \hat{f}(x)=\sum_{1}^{n} f\left(x_{i}\right) .
$$

(b) Si $X$ est réticulé, $\hat{f} \in L_{s}(X)$.

Corollaire 1.3. On suppose que $X$ est réticulé. Soient $f=$ $\sup \left(1_{1}, \cdots, 1_{n}\right)$ avec $1_{1}, \cdots, 1_{n} \in L_{s}(X)$ et $g=\inf \left(1_{1}^{\prime}, \cdots, 1_{m}^{\prime}\right) \quad$ avec $1_{1}^{\prime}, \cdots, 1_{m}^{\prime} \in-L_{s}(X)$. Alors, si $f \leqq g$, on a $\hat{f} \leqq \check{g}$.

Démonstration. Soit $x \in X$. D'après 1.2 (a), il existe $x_{1}, \cdots, x_{n}$, $y_{1} \cdots, y_{m}$ dans $X$ tels que: 


$$
x=\sum_{1}^{n} x_{i}=\sum_{1}^{m} y_{j}
$$

et:

$$
\hat{f}(x)=\sum_{1}^{n} f\left(x_{i}\right) ; \quad \check{g}(x)=\sum_{1}^{m} g\left(y_{i}\right) .
$$

On $\quad a: \hat{f}(x)=\sum_{1}^{n} f\left(x_{i}\right) \leqq \sum_{1}^{n} 1_{j}^{\prime}\left(x_{i}\right)=1_{j}^{\prime}(x)$, pour $1 \leqq j \leqq m$, de sorte que $\hat{f} \leqq g$ et donc, d'après $1.2(\mathrm{~b})$,

$$
\hat{f}(x)=\sum_{1}^{m} \hat{f}\left(y_{i}\right) \leqq \sum_{1}^{m} g\left(y_{j}\right)=\check{g}(x) .
$$

Définition 1.4. On dit qu'un sous-cône convexe $F$ de $X$ est une face de $X$ s'il est héréditaire, en ce sens que:

Si $x, y \in X$ sont tels que $x \leqq y$ et si $y \in F$, alors, $x \in F$.

L'énoncé suivant rappelle les principales proprétés des faces fermées d'un cône réticulé. On trouvera les démonstrations dans [5], 4.17 à 4.20 .

THÉORÈme 1.5. Soit $F$ une face fermée de $X$, supposé réticulé. Alors, (a) Pour tout $x \in X$, l'ensemble $F \cap(x-X)$ possède un unique élément maximal, noté $\pi_{F}(x)$ et l'application $\pi_{F}$ ainsi définie est une application linéaire idempotente de $X$ sur $F$. En particulier, $\pi_{F}$ coïncide avec l'identité sur $F$ et: $X=F+\pi_{F}^{-1}(0)$.

(b) Pour tout $f \in L_{s}(F), f \geqq 0$, l'application $f \circ \pi_{F} \in L S(X)$.

CoRollaine 1.6. Sous les hypothèses du théorème précédent, si $f \in L_{c}(F)$ et $g \in-L_{s}(X)$ et si $g(x) \geqq f(x)$ pour tout $x \in F$, l'application $\varphi$ définie par la formule:

$$
\varphi(x)=f\left(\pi_{F}(x)\right)+g\left(x-\pi_{F}(x)\right),(\forall x \in X)
$$

est dans $-L_{s}(X)$, et elle coïncide avec $f$ sur $F$ et avec $g$ sur $\pi_{F}^{-1}(0)$.

Démonstration. Si on note $g^{\prime}$ la restriction de $g$ à $F$, on $a$ :

$$
\varphi=-\left(g^{\prime}-f\right) \circ \pi_{F}+g .
$$

Ainsi, les propriétés de $\varphi$ résultent immédiatement du théorème précédent.

2. Preliminaires. Les Fréchet ordonnés normaux.

Commencons par rappeler le lemme suivant dont NG, KUNG FU a donné une démonstration élémentaire dans [9]. 
Lemme 2.1. Soient $E$ un e.l.c., $X$ un cône convexe de $E, p$ une semi-norme continue sur $E$ et $E_{1}=\{p \leqq 1\}$. Alors, on $a$ :

$$
\left(E_{1}+X\right) \cap\left(E_{1}-X\right) \subset r E_{1}
$$

si et seulement si:

$$
E_{1}^{\jmath} \subset r . \operatorname{conv}\left(\left(E_{1}^{0} \cap X^{0}\right) \cup-\left(E_{1}^{\jmath} \cap X^{0}\right)\right)
$$

où $r$ est un réel fixé.

Notations 2.2. Soit $E$ un e.l.c. séparé ordonné. On note $E_{+}$ le cône des éléments positifs de $E$. et $H_{+}^{\prime}$ le polaire de $E_{+}$autrement dit le cône des formes linéaires continues et positives sur $E_{+}$. Si $A$ est une partie de $E$, on pose $A_{+}=A \cap E_{+}$et $[A]=$ $\left(A+E_{+}\right) \cap\left(A-E_{+}\right)$.

DÉfInItion 2.3. Soit $E$ un e.l.c. ordonné. On dit qu'une partie $A$ de $E$ est saturée si $[A]=A$. On dit que $E$ est normal s'il existe un sytème fondamental de voisinages de l'origine formé d'ensembles convexes équilibrés saturés.

La notion d'espace normal a été particulièrement étudiée par Schaefer et on trouvera dans [10] $(\mathrm{V}, 3.1)$ cette caractérisation fort commode:

Proposition 2.4. Un e.l.c. ordonné $E$ est normal si et seulement si il existe un système fondamental de semi-normes sur $E$, croissantes sur $E_{+}$.

Exemples de Fréchet normaux 2.5.

(a) Soient $T$ un espace complètement régulier et $V$ une famille dénombrable de fonction s.c.i. sur $T$ à valeurs dans $[0,+\infty)$. On considère l'espace $E_{V}$ des fonctions numériques continues $f$ sur $T$ telles que: $\forall \varepsilon>0, \forall v \in V, \exists K$ compact $\subset T$ tel que: $|f| \leqq \varepsilon v$ hors de $K$. Muni de la topologie définie à partir des semi-normes:

$$
\|f\|_{v}=\inf \{r:|f| \leqq r v\},
$$

$E_{V}$ est un e.l.c. métrisable et normal. Moyennant certaines conditions sur $V$, étudiées en détail dans [6], $E_{V}$ est un Fréchet.

(b) Soient $T$ un espace localement compact localement compact dénombrable à l'infini et $H=\left(H_{n}\right)$ une suite de parties convexes compactes du cône $M_{+}$des mesures de Radon positives sur $T$. On considère l'espace $E_{H}$ des fonctions numériques continues $f$ sur $T$ telles que l'application: $\theta \mapsto \theta(|f|)$ soit continue sur $H_{n}$ pour tout $n$. Mokobodzki et Sibony ont montré que $E_{H}$ est un Fréchet pour la 
topologie de l'ordre et que, pour cette topologie, il existe un système fondamental de semi-normes, croissantes sur $\left(E_{H}\right)_{+}$, (voir [7], Corollaire 22). Ainsi, $E_{H}$ est un Fréchet normal.

(c) Tout sous-espace fermé des espaces $E_{V}$ ou $E_{H}$. Notons que, dans les exemple (a) et (b) toute forme linéaire continue sur $E_{V}$ ou $E_{H}$ se représente au moyen d'une mesure sur l'espace de définition $T$.

Proposition 2.6. Si $E$ est un Fréchet normal à cône positif $E_{+}$ fermé et engendrant, il existe un système fondamental $\left(U_{n}\right)$ de voisinages de l'origine tel que:

(a) $U_{n}+U_{n} \subset U_{n-1}$, $(\forall n \geqq 1)$.

(b) $U_{n}=U_{n+}-U_{n+}$,

(c) $U_{n+}$ est héréditaire,

(d) $\left[U_{n}\right] \subset 3 U_{n}$,

(e) $U_{n}^{0} \subset 3 \operatorname{conv}\left(\left(U_{n}^{0}\right)_{+}-\left(U_{n}^{0}\right)_{+}\right)$,

pour tout entier $n \geqq 0$.

Démonstration. Choisissons un système fondamental $\left(V_{n}\right)$ de voisinages de l'origine formé d'ensembles convexes équilibrés saturés tels que: $V_{n}+V_{n} \subset V_{n-1},\left(\forall_{n} \geqq 1\right)$ et posons:

$$
U_{n}=V_{n+}-V_{n+}\left(\forall_{n} \geqq 0\right) \text {. }
$$

Comme $E$ est un Fréchet et comme $E_{+}$est fermé et engendrant, il résulte d'un théorème de Klee (voir [8], th. 5.3) que la suite $\left(U_{n}\right)$ est un système fondamental de voisinages de l'origine. Cela étant (a) est évident, (b) résulte de ce que $U_{n+}=V_{n+}$, (c) résulte de ce que $V_{n}$ est saturé et (e) se déduit de (d) par le Lemme 2.1. Reste (d). Soit $x \in\left[U_{n}\right]$. Par construction on peut écrire:

$$
x=u-u^{\prime}+e=v-v^{\prime}-e^{\prime} \text {, avec } u, v, u^{\prime}, v^{\prime} \in U_{n+} \text { et } e, e^{\prime} \in E_{+} .
$$

Il en résulte que:

$$
0 \leqq e \leqq v+u^{\prime} .
$$

De (c), on déduit que $e \in 2 U_{n+}$, d'où $x=\left(u-u^{\prime}\right)+e \in 3 U_{n}$

\section{LES THÉORÈMES}

Notations 3.1. Dans la suite de ce travail, $E$ désignera un Fréchet ordonné normal dont le cône positif est fermé ${ }^{1}$ et engendrant et $\left(U_{n}\right)$ un système fondamental de voisinages de l'origine vérifiant les conditions de l'énoncé 2.6. Pour simplifier, on pose:

${ }^{1}$ On notera que cette hypothèse permet de dire qu un élément $x$ de $E$ est positif si et seulement si $L(x) \geqq 0$, pour tout $L$ dans $E_{+}^{\prime}$. 


$$
C_{n}=U_{n}^{0} \cap E_{+}^{\prime}, \quad(\forall n \geqq 0),
$$

et, si $f$ est une fonction numérique définie sur $E_{+}^{\prime}$ (resp. sur $\left.E^{\prime}\right)$ on note:

$$
p_{n}(f)=\sup _{x \in C_{n}}|f(x)|
$$

(resp.

$$
\left.q_{n}(f)=\sup _{x \in U_{n}^{0}}|f(x)|\right)
$$

Lemme 3.2. Si $f$ est une forme linéaire sur $E^{\prime}$, on $a$ :

$$
q_{n}(f) \leqq 3 p_{n}(f), \quad(\forall n \geqq 0) .
$$

Démonstration. C'est une conséquence directe de la Propriété $2.6(\mathrm{e})$.

Lemme 3.3. Pour tout entier $n \geqq 0, C_{n}$ est héréditaire dans $E_{+}^{\prime}$.

Démonstration. Soient $t \in E_{+}^{\prime}, x \in C_{n}$ tels que $0 \leqq t \leqq x$. Pour tout $v \in U_{n+}$, on $a: 0 \leqq t(v) \leqq x(v) \leqq 1$. Pour $v \in U_{n}=U_{n+}-U_{n+}$, on a donc $|t(v)| \leqq 1$, soit $t \in C_{n}$.

Le lien avec les cônes faiblement complets est fourni par le théorème de Klee (voir [8], 5.5) que nous rappelons :

ThÉonème 3.4. Si E est un Fréchet ordonné dont le cône positif est fermé et engendrant, toute forme linéaire positive sur $E$ est continue. Par suite, le cône $E_{+}^{\prime}$ est faiblement complet

Lemme 3.5. Soient $f_{1}, f_{2} \in L_{s}\left(E_{+}^{\prime}\right)$ et $f=\sup \left(f_{1}, f_{2}\right)$. Alors,

$$
p_{n}(\widehat{f}) \leqq 2 p_{n}(f), \quad(\forall n \geqq 0) \text {. }
$$

Démonstration. Soit $x \in C_{n}$. Comme $E_{+}^{\prime}$ est complet, il existe $y, z \in E_{+}^{\prime}$ tels que $x=y+z$ et que $\hat{f}(x)=f(y)+f(z)$. D'après le Lemme 3.3, $y, z \in C_{n}$. Ainsi, $\hat{f}(x) \leqq 2 p_{n}(f)$, d'où l'assertion.

Lemme 3.6. On suppose que $E^{\prime}$ est réticulé et soient $f$ et $-g \in L_{s}\left(E_{+}^{\prime}\right)$ avec $f \leqq g$. Pour tout entier $n \geqq 0$, il existe $h_{1}, h_{2} \in E$ tels que:
(i) $h_{2} \geqq 0$,
(ii) $q_{n}\left(h_{2}\right) \leqq 1$,
(iii) $h_{1} \leqq g$,
(iv) $h_{1}+h_{2} \geqq f$. 
Démonstration. Rappelons d'abord qu'une fonction affine s.c.s. ou s.c.i. sur un convexe compact est bornée (voir [5], 7.3). En particulier, la fonction $f-1 / 6$ est minorée par une constante $c$ sur $C_{n}{ }^{2}$. Cela étant, considérons les ensembles:

$$
\begin{aligned}
& A=\left\{(x, r) \in E^{\prime} x \underline{R}: x \in E_{+}^{\prime} \text { et } r \geqq g(x)\right\} \\
& B=\left\{(x, r) \in E^{\prime} x \underline{R}: x \in C_{n} \text { et } c \leqq r \leqq f(x)-\frac{1}{6}\right\} .
\end{aligned}
$$

Le premier est un cône convexe fermé, le second un convexe compact. Comme ils sont disjoints, il résulte du théorème de HahnBanach qu'il existe une fonction $h_{1} \in E$ telle que:

$$
\begin{aligned}
h_{1}(x) & \leqq g(x) & \left(\forall x \in E_{+}^{\prime}\right) \\
f(x)-\frac{1}{6} & \leqq h_{1}(x) & \left(\forall x \in C_{n}\right) .
\end{aligned}
$$

Posons $f^{\prime}=\sup \left(f-h_{1 \mid E_{+}^{\prime}}, 0\right)$. On $a$ :

$$
0 \leqq f^{\prime}(y)<\frac{1}{6} \quad\left(\forall y \in C_{n}\right)
$$

Ainsi, compte tenu du Lemme 3.3 et de la Proposition 1.2 (a), on $a$ :

$$
0 \leqq \hat{f}^{\prime}(y)<\frac{1}{3}, \quad\left(\forall y \in C_{n}\right) .
$$

Cela étant, considérons les ensembles:

$$
\begin{aligned}
& C=\left\{(x, r) \in E^{\prime} x \underline{R}: x \in E_{+}^{\prime} \text { et } r \leqq \hat{f}^{\prime}(x)\right\} \\
& D=C_{n} x\left\{\frac{1}{3}\right\}
\end{aligned}
$$

L'ensemble $C$ est un cône convexe fermé disjoint du convexe compact $D$. D'après le théorème de Hahn-Banach, il existe $h_{2} \in E$ tel que:

$$
\begin{aligned}
h_{2}(x) & \geqq \hat{f}^{\prime}(x) \geqq 0, \quad\left(\forall x \in E_{+}^{\prime}\right), \\
0 & \leqq h_{2}(y) \leqq \frac{1}{3}, \quad\left(\mathrm{~A} y \in C_{n}\right) .
\end{aligned}
$$

On a donc $p_{n}\left(h_{2}\right) \leqq 1 / 3$, d'où, d'après le Lemme 3.2, $q_{n}\left(h_{2}\right) \leqq 1$. Enfin, on $a$ :

$$
h_{1}(x)+h_{2}(x) \geqq h_{1}(x)+f^{\prime}(x) \geqq f(x), \quad\left(\forall x \in E_{+}^{\prime}\right),
$$

${ }^{2}$ La seule topologie que nous considérons sur $E^{\prime}$ est la topologie faible $\sigma\left(E^{\prime} . E\right)$. 
ce qui achève la démonstration.

ThÉoRÈme 3.7. Soit $E$ un Fréchet ordonné normal à cône positif fermé et engendrant, tel que $E^{\prime}$ soit réticulé et soient $f$ et $-g \in L_{s}\left(E_{+}^{\prime}\right)$ avec $f \leqq g$. Alors, il existe $h \in E$ tel que:

$$
f(x) \leqq h(x) \leqq g(x), \quad\left(\forall x \in E_{+}^{\prime}\right) .
$$

Démonstration. On conserve toujours les notations de 3.1. Nous allons montrer par récurrence qu'il existe deux suites $\left(h_{1}^{n}\right)$ et $\left(h_{2}^{n}\right)$ dans $E$, telles que:

(a) $h_{2}^{n} \geqq 0$,

(b) $h_{2}^{n} \in U_{n}$,

(c) $h_{1}^{n}(x)+h_{2}^{n}(x) \geqq \sup \left(f(x), h_{1}^{n-1}(x)\right),\left(\forall x \in E_{+}^{\prime}\right)$,

(d) $h_{1}^{n+1}(x) \leqq \inf \left(g(x), h_{1}^{n}(x)+h_{2}^{n}(x)\right),\left(\forall x \in E_{+}^{\prime}\right)$.

L'existence de $h_{1}^{0}$ et de $h_{2}^{0}$ découle du lemme précédent appliqué à $n=0$. Supposons trouvé $h_{1}^{p}$ et $h_{2}^{p}$ pour $p \leqq n$. Posons:

$$
\begin{aligned}
f^{\prime} & =\sup \left(f, h_{1 \mid E_{+}^{\prime}}^{n}\right) \text { et } \\
g^{\prime} & =\inf \left(g,\left(h_{1}^{n}+h_{2}^{n}\right)_{\mid E_{+}^{\prime}}\right) .
\end{aligned}
$$

D'après (c) et (d), on a $f^{\prime} \leqq g^{\prime}$. Par suite, comme $E_{+}^{\prime}$ est réticulé et faiblement complet, on $a$, d'après le Corollaire $1.3, \hat{f}^{\prime} \leqq \check{g}^{\prime}$, et d'après la Proposition 1.2, $\hat{f}^{\prime}$ et $-\check{g}^{\prime} \in L_{s}\left(E_{+}^{\prime}\right)$. Le lemme précédent appliqué à ces deux fonctions fournit les fonctions $h_{1}^{n+1}$ et $h_{2}^{n+1}$, ce qui achève le récurrence. Cela étant, on déduit de (b) et de la Propriété 2.6 (a) que la suite $\left(h_{2}^{n}\right)$ est une suite de Cauchy convergeant vers 0 . De $(c)$ et $(d)$, on déduit que:

$$
-h_{2}^{n+1} \leqq h_{1}^{n+1}-h_{1}^{n} \leqq h_{2}^{n} \quad(\forall n \geqq 0),
$$

soit, compte tenu des Propriétés 2.6 (a) et (d),

$$
h_{1}^{n+1}-h_{1}^{n} \in 3 U_{n}, \quad(\forall n \geqq 0),
$$

d'où:

$$
h_{1}^{n+p}-h_{1}^{n} \in 6 U_{n}, \quad(\forall n, p \geqq 0) .
$$

Ainsi, la suite $\left(h_{1}^{n}\right)$ est aussi une suite de Cauchy dans $E$. Soit $h$ sa limite. De (c) et (d), on déduit, en passant à la limite:

$$
f(x) \leqq h(x) \leqq g(x), \quad\left(\forall x \in E_{+}^{\prime}\right) .
$$

Corollaire 3.8. On suppose toujours que $E^{\prime}$ est réticulé. Soient $F$ une face fermée de $E_{+}^{\prime}, \quad l \in L_{c}(F)$ et $f_{1}, \cdots, f_{n}, g_{1}, \cdots, g_{m} \in E$ tels que: 


$$
\begin{aligned}
f_{p} & \leqq g_{q}, \quad(1 \leqq p \leqq n),(1 \leqq q \leqq m) \\
f_{p}(x) & \leqq l(x) \leqq g_{q}(x), \quad(1 \leqq p \leqq n),(1 \leqq q \leqq m),(\forall x \in F)
\end{aligned}
$$

Alors, il existe $h \in E$ avec:

$$
\begin{aligned}
f_{p} & \leqq h \leqq g_{q}, \quad(1 \leqq p \leqq n),(1 \leqq q \leqq m), \\
h(x) & =l(x), \quad(\forall x \in F) .
\end{aligned}
$$

Démonstration. Posons:

$$
f=\sup _{1 \leqq p \leqq n}\left(f_{p \mid E_{+}^{\prime}}\right)^{\wedge} \text { et } g=\inf _{1 \leqq q \leqq m}\left(g_{q \mid E_{+}^{\prime}}\right)^{\vee} \text {. }
$$

Par des arguments déjà vus, on a $f$ et $-g \in L_{s}\left(E_{+}^{\prime}\right)$ et $f \leqq g$. De plus, comme $F$ est une face, il résulte de la Proposition 1.2 (a) que:

$$
f(x) \leqq l(x) \leqq g(x), \quad(\forall x \in F)
$$

Par suite, d'après le Corollaire 1.6, il existe $\varphi$ et $-\psi \in L_{s}\left(E_{+}^{\prime}\right)$ tels que:

$$
\begin{aligned}
& \varphi(y)=\psi(y)=\underline{l}(y), \quad(\forall y \in F) \\
& \varphi(z)=f(z) \text { et } \psi(z)=g(z), \quad\left(\forall z \in \pi_{F}^{-1}(0)\right) .
\end{aligned}
$$

Comme $E_{+}^{\prime}=F+\pi_{F}^{-1}(0)$, d'après le Théorème 1.5 (a), on déduit de (3.8.1), (3.8.2), et (3.8.3) que:

$$
\varphi(x) \leqq \psi(x), \quad\left(\forall x \in E_{+}^{\prime}\right) .
$$

D'après le théorème précédent il existe $h \in E$ tel que:

$$
\varphi(x) \leqq h(x) \leqq \psi(x), \quad\left(\forall x \in E_{+}^{\prime}\right) .
$$

De (3.8.2) résulte que:

$$
h(y)=l(y), \quad(\forall y \in F),
$$

et de (3.8.4) que:

$$
f_{p} \leqq h \leqq g_{q}, \quad(1 \leqq p \leqq n),(1 \leqq q \leqq m) .
$$

CoRollaIRE 3.9. Soit $E$ un Fréchet ordonné normal à cône positif fermé et engendrant. Alors, $E$ vérifie la propriété de décomposition de Riesz si, et seulement si, $E^{\prime}$ la vérifie. Et alors, $E^{\prime}$ est, en fait, réticulé.

Démonstration. Il suffit d'appliquer le corollaire précédent avec $F=\{0\}$ et de remarquer que $E^{\prime}$, qui est positivement engendré 
puisque $E$ est normal (voir 26 (e)), est réticulé dès qu'il vérifie la propriété d'interpolation de Riesz (Prop. 4.15 de [5]).

REMARque 3.10. L'idée de considérer des suites doubles pour montrer le Théorème 3.7 est due à Andô. La démonstration que nous proposons est très voisine de celle de Asimow et Ellis ([2]).

Nous allons appliquer ces résultats à la dualité des idéaux. Dans la suite $E$ désignera un Fréchet normal à cône positif fermé engendrant et qui vérifie la propriété d'interpolation de Riesz.

DÉfinition. 3.11, On dit qu'un sous-espace vectoriel $F$ d'un espace vectoriel ordonné est un idéal d'ordre, ou simplement un idéal si $F_{+}$est une face de $E_{+}$et si $F=F_{+}-F_{+}$.

Lemme 3.12. Si $H$ est un idéal fermé de $E^{\prime}$, son polaire $H^{0}$ dans $E$ est un idéal fermé de $E$.

Démonstration. D'abord, il est clair que $\left(H^{0}\right)_{+}$est une face de $E_{+}$. Il reste à voir que $\left(H^{0}\right)_{+}$engendre $H^{0}$. Autrement dit, que pour tout $f \in H^{0}$, il existe $h \in\left(H^{0}\right)_{+}$avec $h \geqq f$. Fixons $\mathrm{f} \in H^{0}$. Comme $E$ est positivement engendré, il existe $g \in E_{+}$avec $g \geqq f$. On a donc:

$$
\begin{aligned}
& g(x) \geqq \sup (f(x), 0), \quad\left(\forall x \in E_{+}^{\prime}\right) \\
& g(x) \geqq 0 \geqq \sup (f(x), 0), \quad\left(\forall x \in H_{+}\right) .
\end{aligned}
$$

Comme, par hypothèse $H_{+}$est une face de $E_{+}^{\prime}$, il résulte du Corollaire 3.8 qu'il existe $h \in E$, avec:

$$
\begin{aligned}
& h(x)=0, \quad\left(\forall x \in H_{+}\right) \\
& g(x) \geqq h(x) \geqq \sup (f(x), 0), \quad\left(\forall x \in E_{+}^{\prime}\right) .
\end{aligned}
$$

Comme $H=H_{+}-H_{+}$, (i) montre que $h \in H^{0}$ et (ii) montre que $h \in E_{+}$. Ainsi $h \in\left(H^{\circ}\right)_{+}$.

Lemme 3.13. Soient $F$ une face fermée de $E_{+}^{\prime}$ et $C$ une partie convexe héréditaire de $E_{+}^{\prime}$. Alors,

$$
(F-F) \cap \operatorname{conv}(C \cup-C)=\operatorname{conv}((C \cap F) \cup-(C \cap F)) .
$$

Démonstration. On a une inclusion évidente. Inversement, considérons l'application $\pi_{F}$ associée à $F$ dans le Théorème 1.5. Comme $C$ est héréditaire, on a $\pi_{F}(C)=C \cap F$. Par ailleurs, comme $E_{+}^{\prime}$ engendre $E^{\prime}$, on peut prolonger $\pi_{F}$ en une projection de $E^{\prime}$ sur $(F-F)$, qu'on note encore $\pi_{F}$, et on $a$ : 


$$
\begin{gathered}
(F-F) \cap \operatorname{conv}(C \cup-C) \subset \pi_{F}(\operatorname{conv}(C \cup-C) \\
\subset \operatorname{conv}\left(\pi_{F}(C)-\pi_{F}(C)\right)
\end{gathered}
$$

d'où: $(F-F) \cap \operatorname{conv}(C \cup-C) \subset \operatorname{conv}((C \cap F) \cup-(C \cap F))$.

On notera qu'avec une démonstration plus longue, ce résultat est encore vrai si $F$ n'est pas fermée.

THÉORÈmE 3.14. Soit $E$ un Fréchet ordonné normal à cône positif fermé et engendrant. Alors, l'application: $F \rightarrow(F-F)$ est une bijection de l'ensemble des faces fermées de $E_{+}^{\prime}$ sur l'ensemble des idéaux fermés de $E^{\prime}$ et l'application $H \rightarrow H^{0}$ est une bijection de l'ensemble des idéaux fermés de $E^{\prime}$ sur l'ensemble des idéaux fermés de $E$.

Démonstration. Compte tenu du Lemme 3.12, le seul point non évident est de vérifier que $(F-F)$ est fermé pour toute face fermée $F$ de $E_{+}^{\prime}$. Fixons $F$. Choisissons un système fondamental $\left(U_{n}\right)$ de voisinage de l'origine de $E$ comme indiqué en 3.1. Par le lemme précédent,

$$
(F-F) \cap \operatorname{conv}\left(C_{n} \cup-\mathrm{C}_{n}\right)=\operatorname{conv}\left(\left(F \cap C_{n}\right) \cup-\left(F \cap C_{n}\right)\right) .
$$

Ainsi, la trace de $(F-F)$ sur conv $\left(C_{n} \cup-C_{n}\right)$ est fermée. D'après la Propriété 2.6 (e) la trace de $(F-F)$ sur le polaire de chaque voisinage $U_{n}$ est fermée. Par le théorème de Krein-Šsmulian (voir [10], IV, 6.4), $(F-F)$ est fermé dans $E^{\prime}$.

Remarque 3.15. Dans [3], Boboc et Bucur avaient étudié des problèmes voisins de ceux que nous résolvons ici. Ainsi, ils avaient montré les Théorèmes 3.7 et 3.14 dans le cas très particulier où $E_{+}^{\prime}$ était la réunion d'une suite croissante de chapeaux.

\section{REFERENCES}

1. T. Ando, On fundamental properties of a Banach space with a cone, Pacific J. Math. 12, (1962), 1163-1169.

2. L. Asimow and A. J. Ellis, Facial decomposition of linearly compact simplexes and separation of functions on cones, Pacific J. Math., 34, (1970), 301-309.

3. N. Boboc et GH. Bucur, Espaces localement convexes ordonnés. Espaces simpliciaux et leurs duals, Rev. Roum. Math, Pures et Appl., 16, (1971), 449-464.

4. D. A. Edwards, Séparation des fonctions réelles sur un simplexe de Choquet, C. R. Ac. Sci. Paris, 261, (1965), 2798-2800.

5. A. Goullet de Rugy, Géométrie des simplexes, Paris (1968), Centre de Documentation Universitaire et S. E. D. E. S. réunis.

6. - Espaces de fonctions pondérables, Israel J. Math., 12 (2), (1972), 147-160. 7. G. Mokobodzki et D. Sibony, Cones adaptés de fonctions continues et théorie du Potentiel, Séminaire Choquet (Initiation à l'Analyse) $6^{e}$ année (1966/67), nº 5 . 
8. I. Namioka, Partially ordered linear topological spaces, Providence (1957), Mem. Amer. Math. Soc. $\mathrm{n}^{\circ} 24$.

9. NG. KUNG-Fu, On a computation rule for polars, Math. Scand., 26 (1970), 14-16. 10. HH. Schaefer, Topological Vector Spaces, New-York (1966), MacMillan.

Received February 16, 1972.

Université DE PaRIS VI 


\section{PACIFIC JOURNAL OF MATHEMATICS}

\section{EDITORS}

D. Gilbarg and J. Milgram Stanford University

Stanford, California 94305

R. A. Beaumont

University of Washington

Seattle, Washington 98105
J. DugundJI

Department of Mathematics

University of Southern California

Los Angeles, California 90007

RICHARD ARENS

University of California

Los Angeles, California 90024

ASSOCIATE EDITORS
E. F. BECKENBACH
B. H. NeumanN
F. WOLF
K. YoSHIDA

\section{SUPPORTING INSTITUTIONS}

UNIVERSITY OF BRITISH COLUMBIA

CALIFORNIA INSTITUTE OF TECHNOLOGY

UNIVERSITY OF CALIFORNIA

MONTANA STATE UNIVERSITY

UNIVERSITY OF NEVADA

NEW MEXICO STATE UNIVERSITY

OREGON STATE UNIVERSITY

UNIVERSITY OF OREGON

OSAKA UNIVERSITY
UNIVERSITY OF SOUTHERN CALIFORNIA

STANFORD UNIVERSITY

UNIVERSITY OF TOKYO

UNIVERSITY OF UTAH

WASHINGTON STATE UNIVERSITY

UNIVERSITY OF WASHINGTON

$\stackrel{*}{*} \stackrel{*}{*} \stackrel{*}{*}$ AMERICAN MATHEMATICAL SOCIETY

NAVAL WEAPONS CENTER 


\section{Pacific Journal of Mathematics}

\section{Vol. 46, No. $1 \quad$ November, 1973}

Allan Francis Abrahamse, Uniform integrability of derivatives on $\sigma$-lattices .......................................... 1

Ronald Alter and K. K. Kubota, The diophantine equation $x^{2}+D=p^{n} \ldots \ldots \quad 11$

Grahame Bennett, Some inclusion theorems for sequence spaces .......... 17

William Cutler, On extending isotopies ........................ 31

Robert Jay Daverman, Factored codimension one cells in Euclidean

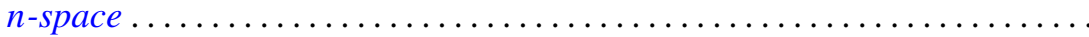

Patrick Barry Eberlein and Barrett O’Neill, Visibility manifolds ............ 45

M. Edelstein, Concerning dentability .......................... 111

Edward Graham Evans, Jr., Krull-Schmidt and cancellation over local

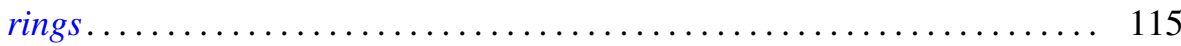

C. D. Feustel, A generalization of Kneser's conjecture ................ 123

Avner Friedman, Uniqueness for the Cauchy problem for degenerate parabolic equations .......................................... 131

David Golber, The cohomological description of a torus action ............ 149

Alain Goullet de Rugy, Un théorème du genre "Andô-Edwards" pour les

Fréchet ordonnés normaux..............................

Louise Hay, The class of recursively enumerable subsets of a recursively enumerable set ........................................

John Paul Helm, Albert Ronald da Silva Meyer and Paul Ruel Young, On orders of translations and enumerations...

Julien O. Hennefeld, A decomposition for $B(X)^{*}$ and unique Hahn-Banach

extensions

Gordon G. Johnson, Moment sequences in Hilbert space .

Thomas Rollin Kramer, A note on countably subparacompact spaces ...

Yves A. Lequain, Differential simplicity and extensions of a derivation ....

Peter Lorimer, A property of the groups Aut $\mathrm{PU}\left(3, q^{2}\right) \ldots$

225

Yasou Matsugu, The Levi problem for a product manifold.

231

John M.F. O'Connell, Real parts of uniform algebras ......

William Lindall Paschke, A factorable Banach algebra without bounded

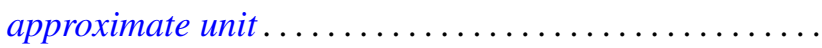

Ronald Joel Rudman, On the fundamental unit of a purely cubic field ....

Tsuan Wu Ting, Torsional rigidities in the elastic-plastic torsion of simply connected cylindrical bars .........................

Philip C. Tonne, Matrix representations for linear transformations on analytic sequences...................................

Jung-Hsien Tsai, On E-compact spaces and generalizations of perfect

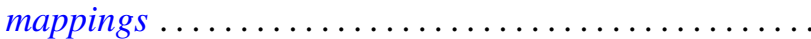

Alfons Van Daele, The upper envelope of invariant functionals majorized by an invariant weight. .. 